\title{
Enhanced growth of anodic alumina nanochannels on Ga-ion pre-irradiated aluminum
}

\author{
C. Y. Liu and A. Datta ${ }^{a)}$ \\ Institute of Atomic and Molecular Sciences, Academia Sinica, P. O. Box 23-166, Taipei 10617, Taiwan \\ N. W. Liu \\ Department of Materials Science and Engineering, National Taiwan University, Taipei 10617, Taiwan \\ Y. R. Wu ${ }^{\text {b) }}$ and H. H. Wang \\ Department of Physics, National Taiwan University, Taipei 10617, Taiwan \\ T. H. Chuang \\ Department of Materials Science and Engineering, National Taiwan University, Taipei 10617, Taiwan \\ Y. L. Wang ${ }^{\mathrm{C})}$ \\ Institute of Atomic and Molecular Sciences, Academia Sinica, P. O. Box 23-166, Taipei 10617, Taiwan and \\ Department of Physics, National Taiwan University, Taipei 10617, Taiwan
}

(Received 19 October 2007; accepted 12 February 2008; published 1 April 2008)

\begin{abstract}
Exposure of an $\mathrm{Al}$ substrate to energetic $\mathrm{Ga}$ ions is found to result in enhanced growth rate of its nanochannels during a subsequent anodization process. Depending on the ion dose, the nanochannels in the pre-irradiated area exhibit different lengths. This interesting phenomenon is exploited by scanning a Ga focused ion beam over the desired area to facilitate the fabrication of arrays of anodic alumina nanochannels with custom-designed super-structure, which is based on the variation of channel length in different areas. (c) 2008 American Vacuum Society.
\end{abstract}

[DOI: $10.1116 / 1.2890706$ ]

\section{INTRODUCTION}

Porous anodic aluminum oxide (AAO) film is widely used as a template for growing various kinds of nanocompopsites because its nanochannels have special properties including high aspect ratio, narrow size distribution, and ordered channel arrangement. ${ }^{1-10}$ To date, the average interchannel spacing has been successfully adjusted from $\sim 10$ to $\sim 500 \mathrm{~nm}$, with the corresponding channel average diameter of $\sim 4$ to $\sim 200 \mathrm{~nm}$, by varying the anodization voltage from 4 to 200 $\mathrm{V} .^{11,12}$ For a given electrolyte, only when the anodization voltage lies within a small range, the nanochannels selforganize into hexagonally closed-packed (HCP) domains. ${ }^{13,14}$ However, the domain size of such nanochannel arrays (simply referred to as self-organized nanochannels hereafter) is limited below a few microns, which presents a serious constraint on the application of AAO. To overcome the limit, an ideally ordered array of nanoholes is artificially patterned on a finely polished $\mathrm{Al}$ foil, and the nanoholes act as pinning points to guide the growth of long-range ordered nanochannels (referred to as guided nanochannels hereafter) in the subsequent anodization process. ${ }^{15-20}$ The size of such nanochannel arrays can be as large as $1 \mathrm{~cm}^{2},{ }^{21}$ and many potential applications are being realized recently. ${ }^{22}$

\footnotetext{
*No corrections received from author prior to publication.

${ }^{a}$ Present address: Amity Institute of Nanotechnology, Amity University, U.P., Sector 125, NOIDA, Gautam Buddha Nagar, Express High Way, NOIDA 201301, India.

${ }^{b)}$ Present address: Graduate Institute of Electro-Optical Engineering, National Taiwan University, Taipei 10617, Taiwan.

${ }^{c}$ Author to whom correspondence should be addressed; electronic mail: ylwang@pub.iams.sinica.edu.tw
}

The guiding nanohole arrays are fabricated by methods that change different physical or chemical properties of the Al. For example, when a Ga focused ion beam (FIB) is employed to fabricate the nanoholes by direct-ion writing, ${ }^{16}$ geometric holes are created by physical sputtering while the chemistry of the $\mathrm{Ga}^{+}$-irradiated area is also changed due to accompanying ion implantation. This work reports that uniform irradiation of $\mathrm{Al}$ by low dose $\mathrm{Ga}$ ion, without creating any significant geometric reliefs on its surface, leads to growth rate enhancement of AAO nanochannels during a subsequent anodization process. By controlling the growth rate variation, nanochannels with different lengths are fabricated on an Al substrate to achieve a super-structured array with custom-designed geometry.

\section{EXPERIMENT}

A high purity (99.99\%) annealed Al-foil $\left(\sim 1 \times 1 \mathrm{~cm}^{2}\right)$ is electro-polished in a mixture of $50 \% \mathrm{HClO}_{4}$ and $\mathrm{C}_{2} \mathrm{H}_{5} \mathrm{OH}$ (volume ratio 1:5) until the root mean square (rms) surface roughness of a typical $10 \times 10 \mu \mathrm{m}^{2}$ area is $\sim 1 \mathrm{~nm}$. A 50 $\mathrm{keV}$ Ga FIB with a beam diameter of $10 \mathrm{~nm}$ and current of $1.1 \mathrm{pA}$ is employed to either deliver uniform ion irradiation or sputter arrays of nanoholes, which have been shown to guide the growth of nanochannels in the subsequent anodization process. The period and depth of the nanoholes arranged in $\mathrm{HCP}$ geometry are 100 and $\sim 3 \mathrm{~nm}$ (corresponding dose of $10^{16}$ ions $\left./ \mathrm{cm}^{2}\right)$, respectively. Anodization of samples is carried out in $0.3 \mathrm{M} \mathrm{H}_{2} \mathrm{C}_{2} \mathrm{O}_{4}$ at $3{ }^{\circ} \mathrm{C}$ with a voltage of $40 \mathrm{~V}$, which leads to the growth $(\sim 3 \mu \mathrm{m} / \mathrm{h})$ of self-organized nanochannel arrays with average interchannel spacing of 100 $\mathrm{nm}$. When needed, the underlying $\mathrm{Al}$ substrate is removed by 


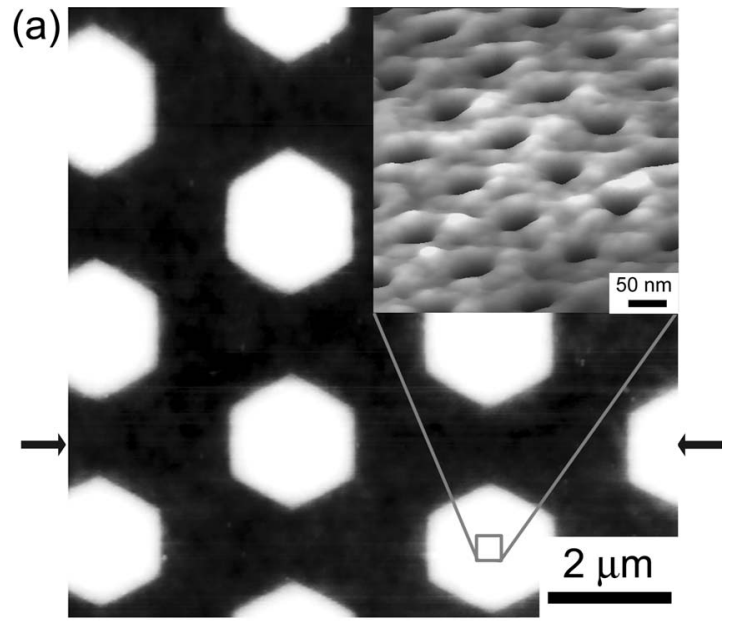

(b)

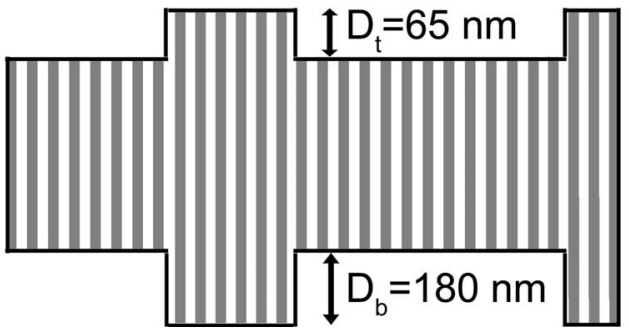

FIG. 1. (a) Top-side AFM image of an AAO film with guided (white regions) and self-organized (dark regions) nanochannels. (Pores are invisible at this scale.) The inset shows a detailed AFM image of the pores $\left(80^{\circ}\right.$ perspective viewing angle). (b) Schematics showing the cross-section of the film in which the guided nanochannels protrude both upward and downward.

a saturated $\mathrm{HgCl}_{2}$ solution at room temperature to gain access to the back-side of the AAO film, and the barrier layer (the continuous oxide layer in the bottom of the nanochannels) is etched in a $5 \% \mathrm{H}_{3} \mathrm{PO}_{4}$ at room temperature to open the nanochannels.

\section{RESULTS AND DISCUSSION}

Figure 1(a) shows an atomic-force-microscope (AFM) image of a $10 \mu \mathrm{m} \mathrm{AAO} \mathrm{film} \mathrm{with} \mathrm{guided} \mathrm{(white} \mathrm{hexagons)}$ and self-organized (surrounding area) arrays of nanochannels. The large black and white contrast in the image reflects the thickness difference between the guided and selforganized areas. The top and bottom of the guided nanochannels are longer by 65 and $180 \mathrm{~nm}$, respectively, as indicated in the schematic of Fig. 1(b). To better understand the growth rate enhancement of the guided nanochannels, a much thinner film of $400 \mathrm{~nm}(10 \mathrm{~min})$ is grown and the length differences are measured. Surprisingly, the results are found to be almost identical to that of the $10 \mu \mathrm{m}$ film $(3 \mathrm{~h})$, indicating the enhanced growth takes place in the first few minutes of the anodization and the guided and self-organized nanochannels grow with the same speed afterward.

The detailed surface topography of the guided nanochannels, as shown in the inset of Fig. 1(a), reveals an interesting effect of the FIB irradiation on the growth of guided nanochannels, which has never been explicitly addressed before. Every guided nanochannel looks like a volcano sitting

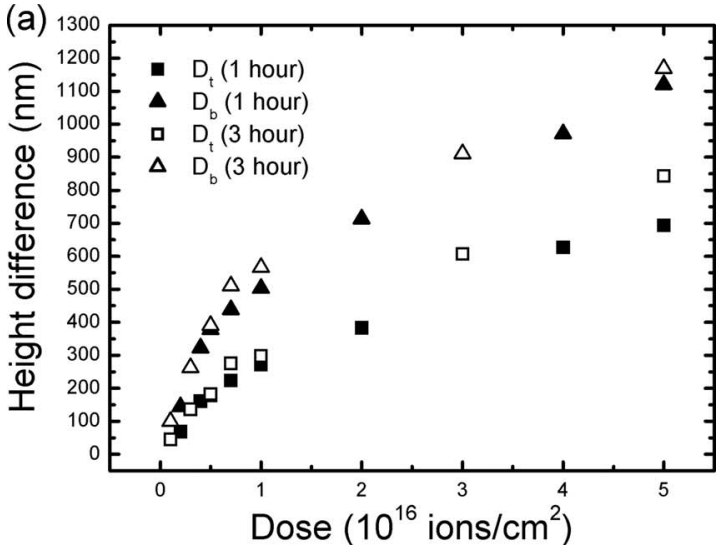

(b)

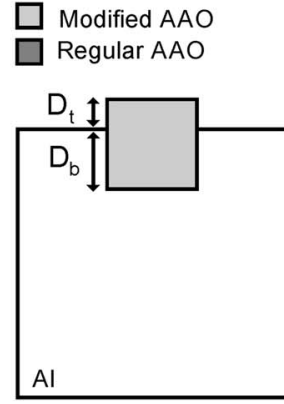

Initial

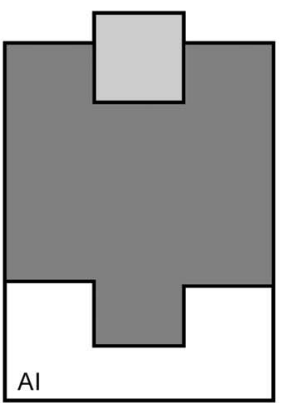

Final

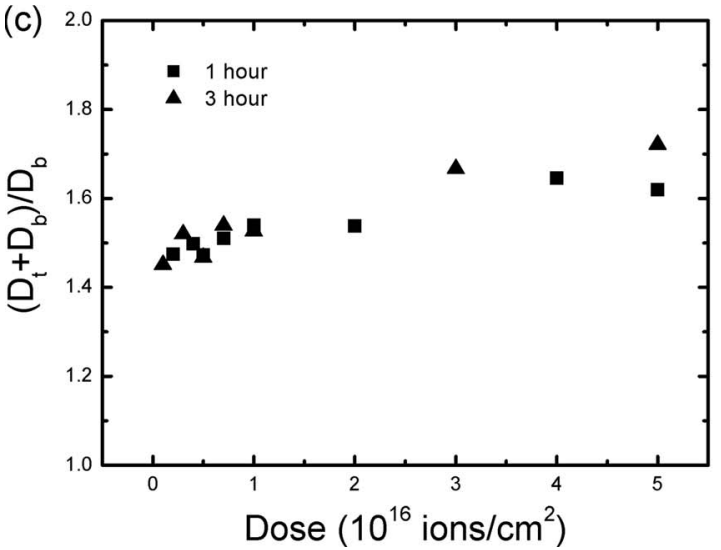

FIG. 2. The top- $\left(D_{t}\right)$ and bottom-side $\left(D_{b}\right)$ height differences between the AAO film grown on $\mathrm{Ga}^{+}$-irradiated and pristine region of an $\mathrm{Al}$ sample as a function of ion dose. Data for anodization times of 3 and $1 \mathrm{~h}$ are included. (b) Schematics showing the cross-sectional profile of the film in the initial and final steps of anodization. Modified AAO refers to the film grown on the $\mathrm{Ga}^{+}$-irradiated area. (c) AAO volume expansion factor, $\left(D_{t}+D_{b}\right) / D_{b}$, as a function of $\mathrm{Ga}^{+}$dose.

on a plateau. Since a guiding nanohole is fabricated by a FIB with a Gaussian profile, the ion dose starts to decrease monotonically from the circumference of the hole. The volcanolike morphology of a guided nanochannel strongly suggests that higher dose of $\mathrm{Ga}^{+}$-irradiation would enhance the rate of $\mathrm{Al}$ anodization.

To verify the postulate, an Al sample is uniformly irradiated with different doses of $\mathrm{Ga}$ and its surface morphology after anodization is measured by AFM. In Fig. 2(a), the top 
$\left(D_{t}\right)$ and bottom $\left(D_{b}\right)$ height differences between the irradiated and neighboring pristine areas on AAO films of different thickness $(10$ and $3 \mu \mathrm{m})$ are plotted as a function of the ion dose. Both $D_{t}$ and $D_{b}$ increase monotonically with the ion dose and become clearly observable for a dose beyond $\sim 1 \times 10^{15}$ ions $/ \mathrm{cm}^{2}$. For a given ion dose, $D_{t}$ and $D_{b}$ of the $10 \mu \mathrm{m}$ film are similar to those of the $3 \mu \mathrm{m}$ film (difference $<10 \%$ ), indicating that the growth rate of the $\mathrm{Ga}^{+}$-irradiated area is enhanced only in the initial anodization step and it becomes essentially the same as that of the pristine area afterward.

The enhanced oxidation caused by $\mathrm{Ga}^{+}$-irradiation has an interesting implication on the volume expansion of the sample during its anodization. As shown by Fig. 2(b), in the initial anodization step, a layer of $\mathrm{Al}$ with a thickness of $D_{b}$ is transformed into AAO with a thickness of $D_{t}+D_{b}$. The volume expansion factor, $\varepsilon=\left(D_{t}+D_{b}\right) / D_{b}$, is found to exhibit clear dependence on the ion dose [Fig. 2(c)]. For our regular AAO, $\varepsilon=1.4$, the same as the reported value, ${ }^{11}$ while for $\mathrm{Al}$ irradiated with $5 \times 10^{16}$ ions $/ \mathrm{cm}^{2}, \varepsilon=1.7$, which is $21 \%$ larger than that of the regular AAO.

We speculate that the abnormally large $\varepsilon$ of AAO formed in a Ga-irradiated area could be related to the Ga-induced spontaneous amalgamation of Al. Woodall et al. found that an Al-Ga alloy ( $\mathrm{Ga}$ atomic percentage $\geq 7 \%$ ) can continuously react with water to produce hydrogen and a muddy alumina. ${ }^{23}$ In a related experiment, a drop of molten Ga was placed on an Al surface and the Ga appeared to penetrate the native aluminum oxide and continued to diffuse into the $\mathrm{Al}$ to form Al-Ga amalgam, which reacted with water to form a muddy alumin. ${ }^{24}$ Similar catalytic effect of Ga could be responsible for the enhanced oxidation of Ga-irradiated Al observed in our experiment. According to TRIM simulation, ${ }^{25}$ the longitudinal range of $\mathrm{Ga}$ in $\mathrm{Al}$ is $35 \mathrm{~nm}$. Exposed to a dose of $5 \times 10^{16}$ ions $/ \mathrm{cm}^{2}$, the atomic percentage of $\mathrm{Ga}$ in Al could reach 9\%, higher than that used in Woodall's experiment. Therefore, our speculation is justifiable.

Although the mechanism of AAO growth-enhancement induced by either uniform $\mathrm{Ga}^{+}$-irradiation or FIB prepatterning of nanoholes is yet to be clearly understood, these techniques can be employed jointly to fabricate an AAO film consisting of nanochannels with different lengths. For example, four kinds of nanochannel array can be grown simultaneously on an AAO film, as indicated in its optical microscope image [Fig. 3(a)]. Area A is a pristine Al surface and therefore forms self-organized nanochannels after anodization. Area B is exposed to a uniform dose of $10^{15}$ ions $/ \mathrm{cm}^{2}$ and therefore forms self-organized nanochannels longer than that in area A. Additional FIB lithography is conducted on areas $\mathrm{C}$ and $\mathrm{D}$ to fabricate arrays of guiding nanoholes using pixel dwell times of 6 and $12 \mathrm{~ms}$, respectively. The guided nanochannels grown in area $\mathrm{C}$ are therefore longer than the self-organized nanochannels in B. Because of the longer FIB dwell time, the guided nanochannels in $\mathrm{D}$ are the longest, as shown by the top and bottom AFM line profiles in Fig. 3(b). The example clearly demonstrates that the length of both self-organized and guided nanochannels on an AAO film can
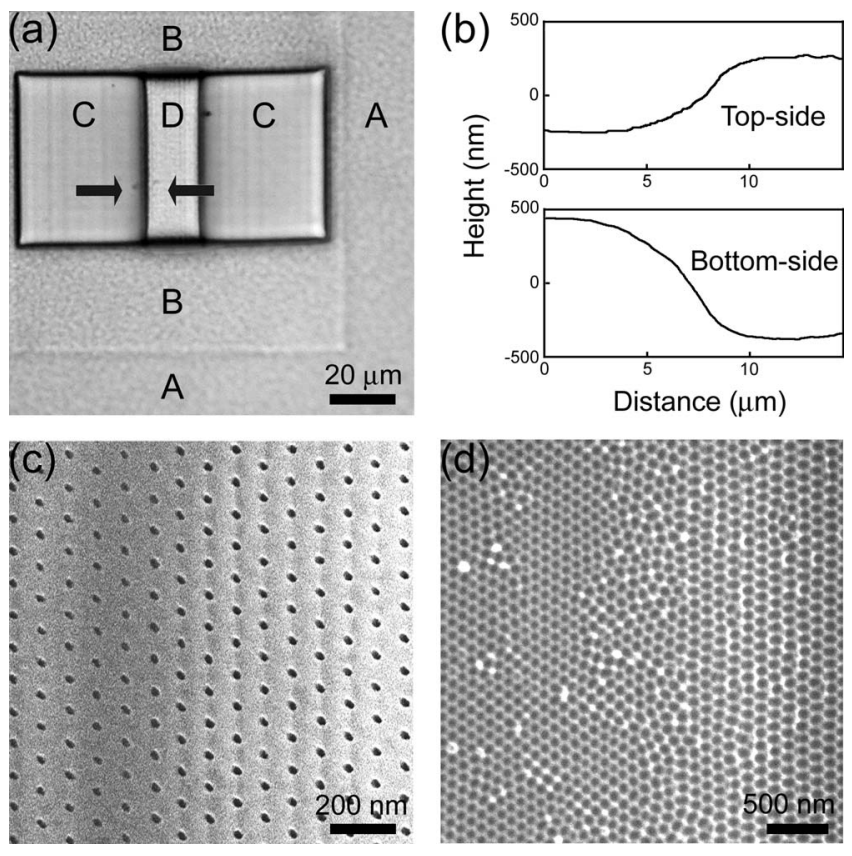

FIG. 3. (a) Top view optical microscope image of an AAO film (5 min anodization time) with self-organized ( $\mathrm{A}$ and $\mathrm{B})$ and guided (C and $\mathrm{D})$ nanochannels. The thickness differences, (top+bottom), between the film on $\mathrm{A}$ and $\mathrm{B}, \mathrm{B}$ and $\mathrm{C}$, and $\mathrm{C}$ and $\mathrm{D}$ are $(50+100) \mathrm{nm},(400+700) \mathrm{nm}$, and $(500+800) \mathrm{nm}$, respectively. (b) Top-side and bottom-side AFM line profiles along the line indicated by arrows in (a). (c) Top-side and (d) bottom-side SEM image of nanochannels on the boundary between areas $\mathrm{C}$ (left part) and $\mathrm{D}$ (right part)

be locally varied by a combination of uniform ion-irradiation and FIB prepatterning using different ion doses.

Creation of length difference between two neighboring arrays of guided nanochannel by uniform ion-irradiation always leads to the formation of a transitional region with slanted surface profile, as shown in Fig. 3(b). The top of the nanochannels in the transitional region is perfectly ordered while the bottom is not as ordered, as indicated by the scanning electron microscope (SEM) images in Figs. 3(c) and $3(\mathrm{~d})$, respectively. Because the boundary area is a slanted surface, the stress on the each nanochannel in this region is hard to balance; ${ }^{26}$ we speculate the unbalanced stress is the driving force for gradually destroying the order of an array as it grows longer. The presence of the slanted surface put a limit on the edge definition of this lithographic method. By simply measuring the slopes of the line profiles in Fig. 3(b), the top and bottom edge definitions, $\Delta x_{t}$ and $\Delta x_{b}$, are estimated as $\Delta x_{t} \sim 9 D_{t}$ and $\Delta x_{b} \sim 6 D_{b}$.

Given the above limit on edge definition, if $\Delta x_{t}$ of few channel-spacing $(100 \mathrm{~nm})$ is desired, the maximum of $D_{t}$ is on the order of a few tens of nanometers. Such a surface relief is adequate for a stamp used in nano-imprint. ${ }^{27-29} \mathrm{As}$ an example, Fig. 4 shows a super-structured AAO fabricated by a combination of guided nanochannel growth and uniform $\mathrm{Ga}^{+}$-irradiation. Both the longer (orange) and shorter (blue) nanochannels are guided and therefore ordered. In principle, two different materials can be loaded into two types of nanochannels respectively and printed onto a substrate to 


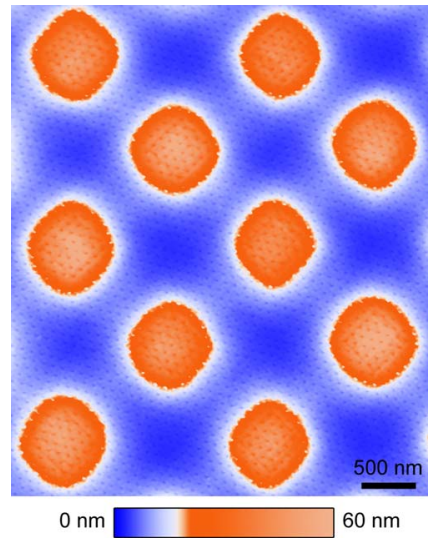

FIG. 4. AFM image of the top-side of an AAO film with super-structured array of guided nanochannels. The nanochannels in the square areas (orange) are longer because the regions on the Al surface are uniformly exposed to an additional dose of $2 \times 10^{15}$ ions $/ \mathrm{cm}^{2}$ before anodization.

form a 2D super-lattice of nanodots. It should also be noted that AAO nanochannel arrays with much more complex super-structure, such as multi-level of channel length, can be easily achieved by irradiating different area with different ion dose; and such an array can be used to form a much more complicated 2D super-lattice of nanodots. Since the interchannel spacing, the size, and the geometry of the $\mathrm{Ga}^{+}$-irradiated areas can be varied easily in our fabrication process, it facilitates the fabrication of a new class of stamps for nano-imprint.

\section{CONCLUSION}

Uniform pre-irradiation of $\mathrm{Al}$ with $\mathrm{Ga}$ ions enhances the growth rate of anodic alumina nanochannels. In conjunction with FIB lithography, the growth rate enhancement has been exploited to fabricate arrays of guided or self-organized nanochannels with different lengths arranged in a customdesigned geometry. The technique opens the possibility of fabricating arrays of super-structured nanochannels, which may find applications in various nanofabrication technologies including for nano-imprint lithography.

\section{ACKNOWLEDGMENTS}

This work was partly funded by the National Science Council of Taiwan (Grant No. NSC96-2120-M-001-002). The authors acknowledge discussion and help from J. K. Wang, M. Y. Lai, H. H. Chang, and S. B. Wu. C.Y.L. ac- knowledges the fellowship provided by Academia Sinica, Taiwan.

${ }^{1}$ H. Masuda and K. Fukuda, Science 268, 1466 (1995).

${ }^{2}$ T. G. Tsai, K. J. Chao, X. J. Guo, S. L. Sung, C. N. Wu, Y. L. Wang, and H. C. Shih, Adv. Mater. (Weinheim, Ger.) 9, 1154 (1997).

${ }^{3}$ A. P. Li, F. Müller, A. Bimer, K. Nielsch, and U. Gösele, Adv. Mater. (Weinheim, Ger.) 11, 483 (1999).

${ }^{4} \mathrm{~J}$. Li, C. Papadopoulos, and J. Xu, Nature (London) 402, 253 (1999).

${ }^{5}$ C. Papadopoulos, A. Rakitin, J. Li, A. S. Vedeneev, and J. M. Xu, Phys. Rev. Lett. 85, 3476 (2000)

${ }^{6}$ E. J. Bae, W. B. Choi, K. S. Jeong, J. U. Chu, G. S. Park, S. Song, and I. K. Yoo, Adv. Mater. (Weinheim, Ger.) 14, 277 (2002).

${ }^{7}$ R. Z. Chen, D. S. Xu, G. L. Guo, and L. L. Gui, J. Mater. Chem. 12, 2435 (2002).

${ }^{8}$ G. Sauer, G. Brehm, S. Schneider, K. Nielsch, R. B. Wehrspohn, J. Choi, H. Hofmeister, and U. Gösele, J. Appl. Phys. 91, 3243 (2002).

${ }^{9}$ M. N. Lin, C. Y. Liu, N. W. Liu, M. Y. Lai, C. Y. Peng, H. H. Wang, Y. L. Wang, and M. T. Lin, Nanotechnology 17, 315 (2006).

${ }^{10}$ H. H. Wang, C. Y. Liu, S. B. Wu, N. W. Liu, C. Y. Peng, T. H. Chan, C. F. Hsu, J. W. Wang, and Y. L. Wang, Adv. Mater. (Weinheim, Ger.) 18, 491 (2006).

${ }^{11}$ A. P. Li, F. Müller, A. Birner, K. Nielsch, and U. Gösele, J. Appl. Phys. 84, 6023 (1998).

${ }^{12}$ H. D. L. Lira and R. Paterson, J. Membr. Sci. 206, 375 (2002).

${ }^{13}$ O. Jessensky, F. Müller, and U. Gösele, Appl. Phys. Lett. 72, 1173 (1998).

${ }^{14}$ F. Li, L. Zhang, and R. M. Metzger, Chem. Mater. 10, 2470 (1998).

${ }^{15}$ H. Masuda, H. Yamada, M. Satoh, and H. Asoh, Appl. Phys. Lett. 71, 2770 (1997).

${ }^{16}$ C. Y. Liu, A. Datta, and Y. L. Wang, Appl. Phys. Lett. 78, 120 (2001).

${ }^{17}$ Z. Sun and H. K. Kima, Appl. Phys. Lett. 81, 3458 (2002).

${ }^{18}$ N. W. Liu, A. Datta, C. Y. Liu, and Y. L. Wang, Appl. Phys. Lett. 82, 1281 (2003).

${ }^{19}$ S. Fournier-Bidoz, V. Kitaev, D. Routkevitch, I. Manners, and G. A. Ozin, Adv. Mater. (Weinheim, Ger.) 16, 2193 (2004).

${ }^{20}$ N. W. Liu, A. Datta, C. Y. Liu, C. Y. Peng, H. H. Wang, and Y. L. Wang, Adv. Mater. (Weinheim, Ger.) 17, 222 (2005).

${ }^{21}$ J. Choi, K. Nielsch, M. Reiche, R. B. Wehrspohn, and U. Gösele, J. Vac. Sci. Technol. B 21, 763 (2003).

${ }^{22}$ H. Oshima, H. Kikuchi, H. Nakao, K. I. Itoh, T. Kamimura, T. Morikawa, K. Matsumoto, T. Umada, H. Tamura, K. Nishio, and H. Masuda, Appl. Phys. Lett. 91, 022508 (2007).

${ }^{23}$ J. M. Woodall, Making of Hydrogen from Aluminum, http:// hydrogen.ecn.purdue.edu/.

${ }^{24}$ D. O. Flamini, S. B. Saidman, and J. B. Bessone, Corros. Sci. 48, 1413 (2006).

${ }^{25}$ J. F. Zeigler and J. P.' Biersack, SRIM (TRIM 90) Simulation package, IBM Research, 28-024 Yorktown, NY, 10598 (1995).

${ }^{26}$ C. Y. Liu, A. Datta, N. W. Liu, C. Y. Peng, and Y. L. Wang, Appl. Phys. Lett. 84, 2509 (2004).

${ }^{27}$ C. M. S. Sotomayor Torres, S. Zankovych, J. Seekamp, A. P. Kam, C. C. Cedeño, T. Hoffmann, J. Ahopelto, F. Reuther, K. Pfeiffer, G. Bleidiessel, G. Gruetzner, M. V. Maximov, and B. Heidari, Mater. Sci. Eng., C 23, 23 (2003).

${ }^{28}$ H. H. Lee, K. S. Chou, and K. C. Huang, Nanotechnology 16, 2436 (2005).

${ }^{29}$ H. S. Koo, M. Chen, P. C. Pan, L. T. Chou, F. M. Wu, S. J. Chang, and T. Kawai, Displays 27, 124 (2006). 